\title{
A Variational Principle for Three-Point Boundary Value Problems with Impulse
}

\author{
Dianwu Yang \\ School of Mathematical Sciences, University of Jinan, Jinan 250022, China \\ Correspondence should be addressed to Dianwu Yang; ss_yangdw@ujn.edu.cn
}

Received 20 January 2014; Accepted 11 March 2014; Published 10 April 2014

Academic Editor: Shurong Sun

Copyright (C) 2014 Dianwu Yang. This is an open access article distributed under the Creative Commons Attribution License, which permits unrestricted use, distribution, and reproduction in any medium, provided the original work is properly cited.

We construct a variational functional of a class of three-point boundary value problems with impulse. Using the critical points theory, we study the existence of solutions to second-order three-point boundary value problems with impulse.

\section{Introduction}

In this paper, we study the following three-point boundary value problems with impulse:

$$
\begin{aligned}
x^{\prime \prime} & =f(t, x), \quad t \neq t_{1}, t \in[0,1], \\
x^{\prime}(0) & =a_{11} x(0)+a_{12} x\left(t_{1}\right)+a_{13} x(1), \\
\Delta x^{\prime}\left(t_{1}\right) & =a_{12} x(0)+a_{22} x\left(t_{1}\right)+a_{23} x(1), \\
x^{\prime}(1) & =-a_{13} x(0)-a_{23} x\left(t_{1}\right)-a_{33} x(1),
\end{aligned}
$$

where $0<t_{1}<1, f:[0,1] \times R \rightarrow R, \Delta x^{\prime}\left(t_{1}\right)=x^{\prime}\left(t_{1}^{+}\right)-$ $x^{\prime}\left(t_{1}^{-}\right)$, and $x^{\prime}\left(t_{1}^{+}\right)$(respectively, $x^{\prime}\left(t_{1}^{-}\right)$) denote the right limit (respectively, left limit) of $x^{\prime}(t)$ at $t_{1}$.

The existence of solutions for three-point boundary value problems has been investigated by many authors. See, for example, [1-11] and references cited therein. In [1], Bao et al. studied a class of three-point boundary value problems

$$
\begin{gathered}
y^{\prime \prime}(t)+f\left(t, y(t), y^{\prime}(t)\right)=0, \quad 0<t<1, \\
y(0)=0, \quad y(1)=\gamma y(\eta) .
\end{gathered}
$$

Using the method upper and lower solutions, some existence results for positive solutions of problems (2) had been obtained. By applying the fixed point theory, many authors have studied the existence of positive solutions for threepoint boundary value problems (see [7-11]). In [12], the authors studied Sturm-Liouville boundary value problem of a class of second-order impulsive differential equations:

$$
\begin{aligned}
&-u^{\prime \prime}(t)+u(t)=f(t, u(t)), \quad t \in[0, T] \backslash\left\{t_{1}, t_{2}, \ldots, t_{p}\right\}, \\
&-\Delta u\left(t_{j}\right)=A_{j} u\left(t_{j}^{-}\right), \quad j=1,2, \ldots, p, \\
&-\Delta u^{\prime}\left(t_{j}\right)=I_{j}\left(u\left(t_{j}^{-}\right)\right)-A_{j} u^{\prime}\left(t_{j}^{+}\right), \quad j=1,2, \ldots, p, \\
& \alpha u(0)-\beta u^{\prime}(0)=0, \quad \gamma u(T)+\sigma u^{\prime}(T)=0 .
\end{aligned}
$$

By establishing the corresponding variational principle of problem (3), the existence for solutions of the problems had been obtained. In papers [12-16], the variational methods were applied to impulsive differential equations too. In this paper, we will use the critical point theorem to study threepoint boundary value problems (1) with impulse.

The paper is organized as follows: in Section 2, we will construct a variational functional of the boundary value problem (1). In Section 3, using the critical point theory, we will give some sufficient conditions in which problem (1) has solutions. Throughout this paper, we always assume that the following conditions hold.

$\left(A_{1}\right) f(t, x)$ is measurable in $t$ for each $x \in R$, continuous in $x$ for almost every $t \in[0,1]$.

$\left(A_{2}\right)$ for any $k>0$, there exists $h_{k} \in L^{1}(0,1)$ such that

$$
|f(t, x)| \leq h_{k}(t)
$$

for almost every $t \in[0,1]$ and for all $|x| \leq k$. 


\section{Variational Structure}

Let $W$ be the space of absolutely continuous functions $x$ : $[0,1] \rightarrow R$ with a weak derivative $x^{\prime} \in L^{2}(0,1 ; R)$. We define the operator $P$ on $W$ by

$$
(P x)(t)= \begin{cases}\frac{x\left(t_{1}\right)-x(0)}{t_{1}}\left(t-t_{1}\right)+x\left(t_{1}\right), & 0 \leq t \leq t_{1}, \\ \frac{x(1)-x\left(t_{1}\right)}{1-t_{1}}\left(t-t_{1}\right)+x\left(t_{1}\right), & t_{1}<t \leq 1,\end{cases}
$$

and set $W_{1}=P W$ and $W_{2}=(I-P) W$. The following properties are easy consequence of the definition:

$\left(B_{1}\right) \operatorname{dim} W_{1}=3$.

$\left(B_{2}\right) W=W_{1} \oplus W_{2}$.

$\left(B_{3}\right)$ For each $x \in W_{2}, x(0)=x\left(t_{1}\right)=x(1)=0$.

Now, we define the norm $\|\cdot\|$ over $W$ by

$$
\|x\|^{2}=\int_{0}^{1}\left[((I-P) x)^{\prime}(t)\right]^{2} d t+x^{2}(0)+x^{2}\left(t_{1}\right)+x^{2}(1) .
$$

Then $W$ is a Hilbert space and the corresponding inner product $(x, y)$ is

$$
\begin{aligned}
(x, y)= & \int_{0}^{1}\left[((I-P) x)^{\prime}(t) \cdot((I-P) y)^{\prime}(t)\right] d t \\
& +x(0) y(0)+x\left(t_{1}\right) y\left(t_{1}\right)+x(1) y(1) .
\end{aligned}
$$

For each $x \in W$, it follows from (6) that

$$
\begin{aligned}
x^{2}(t) & =\left[x(0)+\int_{0}^{t} x^{\prime}(s) d s\right]^{2} \\
& =\left[x(1)+\int_{0}^{t}((I-P) x)^{\prime}(s) d s\right]^{2} \\
& \leq 2 x^{2}(1)+2 \int_{0}^{1}\left[((I-P) x)^{\prime}(t)\right]^{2} d t \leq 2\|x\|^{2},
\end{aligned}
$$

and so $|x(t)| \leq \sqrt{2}\|x\|$ on $[0,1]$.

In order to study problem (1), we define the functional $\phi$ on $W$ by

$$
\begin{aligned}
\phi(x)= & \frac{1}{2}\|(I-P) x\|^{2}+\int_{0}^{1} F(t, x(t)) d t \\
& +\frac{1}{2}\left(x(0), x\left(t_{1}\right), x(1)\right) A\left(x(0), x\left(t_{1}\right), x(1)\right)^{T},
\end{aligned}
$$

where $F(t, x)=\int_{0}^{x} f(t, u) d u$ and

$$
A=\left(\begin{array}{ccc}
a_{11}+\frac{1}{t_{1}} & a_{12}-\frac{1}{t_{1}} & a_{13} \\
a_{12}-\frac{1}{t_{1}} & a_{22}+\frac{1}{t_{1}}+\frac{1}{1-t_{1}} & a_{23}-\frac{1}{1-t_{1}} \\
a_{13} & a_{23}-\frac{1}{1-t_{1}} & a_{33}+\frac{1}{1-t_{1}}
\end{array}\right) .
$$

Under the conditions $\left(A_{1}\right)$ and $\left(A_{2}\right), \phi$ is continuously differentiable, weakly lower semicontinuous on $W$ and

$$
\begin{aligned}
\left(\phi^{\prime}(x), y\right)= & ((I-P) x,(I-P) y) \\
& +\int_{0}^{1} f(t, x(t)) y(t) d t \\
& +\left(x(0), x\left(t_{1}\right), x(1)\right) A\left(y(0), y\left(t_{1}\right), y(1)\right)^{T}
\end{aligned}
$$

for all $y \in W$; see [17].

The following theorem is the main conclusion of this paper.

Theorem 1. Assume that $f$ satisfies the conditions $\left(A_{1}\right)$ and $\left(A_{2}\right)$. If $x$ is a critical point of the functional $\phi$ defined by (9), then $x(t)$ is a solution of problem (1).

Proof. Let $x$ be a critical point of the functional $\phi$ defined by (9). We prove this theorem in three steps.

Step 1. In this step, we prove that $x(t)$ satisfies the equation $x^{\prime \prime}(t)=f(t, x(t))$ except at $t_{1}$.

We define $\omega \in C(0,1 ; R)$ by

$$
\omega(t)=\int_{t_{1}}^{t} f(s, x(s)) d s .
$$

It follows from (11) that for all $y \in W$,

$$
\begin{aligned}
& ((I-P) x,(I-P) y)+\int_{0}^{1} f(t, x(t)) y(t) d t \\
& \quad+\left(x(0), x\left(t_{1}\right), x(1)\right) A\left(y(0), y\left(t_{1}\right), y(1)\right)^{T}=0 .
\end{aligned}
$$

By the Fubini theorem and (13), we obtain

$$
\begin{aligned}
\int_{0}^{1}\left[((I-P) x)^{\prime}(t)-\omega(t)\right] y^{\prime}(t) d t \\
=\int_{0}^{1}((I-P) x)^{\prime}(t)\left[((I-P) y)^{\prime}(t)+(P y)^{\prime}(t)\right] d t \\
\quad-\int_{0}^{1} \omega(t) y^{\prime}(t) d t \\
=-\int_{0}^{1} f(t, x(t)) y(t) d t \\
\quad-\left(x(0), x\left(t_{1}\right), x(1)\right) A\left(y(0), y\left(t_{1}\right), y(1)\right)^{T} \\
\quad-\int_{0}^{1} y^{\prime}(t) \int_{t_{1}}^{t} f(s, x(s)) d s d t \\
=-y(0) \int_{0}^{t_{1}} f(t, x(t)) d t-y(1) \int_{t_{1}}^{1} f(t, x(t)) d t \\
\quad-\left(x(0), x\left(t_{1}\right), x(1)\right) A\left(y(0), y\left(t_{1}\right), y(1)\right)^{T} .
\end{aligned}
$$


In particular, we can choose

$$
\begin{aligned}
& y(t)= \begin{cases}\sin \frac{2 n \pi t}{t_{1}} & 0 \leq t \leq t_{1}, \quad n=1,2, \ldots, \\
0 & t_{1}<t \leq 1,\end{cases} \\
& y(t)=\left\{\begin{array}{ll}
1-\cos \frac{2 n \pi t}{t_{1}} & 0 \leq t \leq t_{1}, \\
0 & t_{1}<t \leq 1,
\end{array} \quad n=1,2, \ldots,\right.
\end{aligned}
$$

so that

$$
\begin{array}{r}
\int_{0}^{t_{1}}\left[((I-P) x)^{\prime}(t)-\omega(t)\right] \sin \frac{2 n \pi t}{t_{1}} d t \\
=\int_{0}^{t_{1}}\left[((I-P) x)^{\prime}(t)-\omega(t)\right] \cos \frac{2 n \pi t}{t_{1}} d t=0, \\
n=1,2, \ldots
\end{array}
$$

The theorem of Fourier series implies that

$$
((I-P) x)^{\prime}(t)-\omega(t)=C
$$

on $\left[0, t_{1}\right]$ for some $C \in R$. Integrating (17) over $\left[0, t_{1}\right]$, we obtain

$$
C t_{1}=-\int_{0}^{t_{1}} \omega(t) d t=\int_{0}^{t_{1}} t f(t, x(t)) d t,
$$

and hence

$$
((I-P) x)^{\prime}(t)-\omega(t)=\int_{0}^{t_{1}} \frac{t}{t_{1}} f(t, x(t)) d t .
$$

Similarly, setting

$$
\begin{aligned}
& y(t)=\left\{\begin{array}{ll}
0 & 0 \leq t \leq t_{1}, \\
\sin \frac{2 n \pi\left(t-t_{1}\right)}{1-t_{1}} & t_{1}<t \leq 1,
\end{array} \quad n=1,2, \ldots,\right. \\
& y(t)=\left\{\begin{array}{ll}
0 & 0 \leq t \leq t_{1}, \\
1-\cos \frac{2 n \pi\left(t-t_{1}\right)}{1-t_{1}} & t_{1}<t \leq 1,
\end{array} \quad n=1,2, \ldots,\right.
\end{aligned}
$$

in (14), we have

$$
((I-P) x)^{\prime}(t)-\omega(t)=-\int_{t_{1}}^{1} \frac{1-t}{1-t_{1}} f(t, x(t)) d t
$$

on $\left[t_{1}, 1\right]$. Thus, (19) and (21) imply that $x(t)$ satisfies the equation $x^{\prime \prime}(t)=f(t, x(t))$ except at $t_{1}$.

Step 2. In this step, we prove that $x(t)$ satisfies the boundary value conditions $x^{\prime}(0)=a_{11} x(0)+a_{12} x\left(t_{1}\right)+a_{13} x(1)$ and $x^{\prime}(1)=-a_{13} x(0)-a_{23} x\left(t_{1}\right)-a_{33} x(1)$.

Set

$$
y(t)= \begin{cases}\frac{t}{t_{1}}-1 & 0 \leq t \leq t_{1} \\ 0 & t_{1}<t \leq 1\end{cases}
$$

Inserting (22) into (13), we have

$$
\begin{aligned}
& \int_{0}^{t_{1}}\left(\frac{t}{t_{1}}-1\right) f(t, x(t)) d t \\
& \quad=\left(a_{11}+\frac{1}{t_{1}}\right) x(0)+\left(a_{12}-\frac{1}{t_{1}}\right) x\left(t_{1}\right)+a_{13} x(1) .
\end{aligned}
$$

It follows form (19) and (23) that

$$
\begin{aligned}
x^{\prime}(0) & =((I-P) x)^{\prime}(0)+(P x)^{\prime}(0) \\
& =\int_{0}^{t_{1}}\left(\frac{t}{t_{1}}-1\right) f(t, x(t)) d t+\frac{x\left(t_{1}\right)-x(0)}{t_{1}} \\
& =a_{11} x(0)+a_{12} x\left(t_{1}\right)+a_{13} x(1) .
\end{aligned}
$$

Similarly, setting

$$
y(t)= \begin{cases}0 & 0 \leq t \leq t_{1} \\ \frac{t-t_{1}}{1-t_{1}} & t_{1}<t \leq 1\end{cases}
$$

equality (13) becomes

$$
\begin{aligned}
& \int_{t_{1}}^{1} \frac{t-t_{1}}{1-t_{1}} f(t, x(t)) d t+a_{13} x(0) \\
& \quad+\left(a_{23}-\frac{1}{1-t_{1}}\right) x\left(t_{1}\right)+\left(a_{33}+\frac{1}{1-t_{1}}\right) x(1)=0,
\end{aligned}
$$

and hence

$$
\begin{aligned}
x^{\prime}(1) & =((I-P) x)^{\prime}(1)+(P x)^{\prime}(1) \\
& =\int_{t_{1}}^{1} \frac{t-t_{1}}{1-t_{1}} f(t, x(t)) d t+\frac{x(1)-x\left(t_{1}\right)}{1-t_{1}} \\
& =-a_{13} x(0)-a_{23} x\left(t_{1}\right)-a_{33} x(1) .
\end{aligned}
$$

Step 3. In this step, we prove that $x(t)$ satisfies the conditions $\Delta x^{\prime}\left(t_{1}\right)=a_{12} x(0)+a_{22} x\left(t_{1}\right)+a_{23} x(1)$.

Inserting

$$
y(t)= \begin{cases}\frac{t}{t_{1}} & 0 \leq t \leq t_{1} \\ \frac{1-t}{1-t_{1}} & t_{1}<t \leq 1\end{cases}
$$

into (13), we have

$$
\begin{aligned}
& -\int_{0}^{t_{1}} \frac{t}{t_{1}} f(t, x(t)) d t-\int_{t_{1}}^{1} \frac{1-t}{1-t_{1}} f(t, x(t)) d t \\
& \quad=\left(a_{12}-\frac{1}{t_{1}}\right) x(0)+\left(a_{22}+\frac{1}{t_{1}}+\frac{1}{1-t_{1}}\right) x\left(t_{1}\right) \\
& \quad+\left(a_{23}-\frac{1}{1-t_{1}}\right) x(1) .
\end{aligned}
$$


It follows from (19) and (21) that

$$
\begin{aligned}
\Delta x^{\prime}\left(t_{1}\right)= & \Delta((I-P) x)^{\prime}\left(t_{1}\right)+\Delta(P x)^{\prime}\left(t_{1}\right) \\
= & -\int_{0}^{t_{1}} \frac{t}{t_{1}} f(t, x(t)) d t-\int_{t_{1}}^{1} \frac{1-t}{1-t_{1}} f(t, x(t)) d t \\
& +\frac{x(1)-x\left(t_{1}\right)}{1-t_{1}}-\frac{x\left(t_{1}\right)-x(0)}{t_{1}} \\
= & a_{12} x(0)+a_{22} x\left(t_{1}\right)+a_{23} x(1) .
\end{aligned}
$$

This completes the proof of Theorem 1 .

\section{Solutions of Problem (1)}

As applications of Theorem 1, we consider solutions of problem (1). Let $k_{1}$ and $k_{2}$ denote the minimum and maximum eigenvalue of the matrix $A$ in (9). We have the following theorems.

Theorem 2. Assume that $f$ satisfies $\left(A_{1}\right)$ and $\left(A_{2}\right)$. Assume also that the following conditions hold:

$\left(A_{3}\right) k_{1}>0$.

$\left(A_{4}\right)$ there is a positive constant $l$, with $l<2$, and a positive function $c \in L^{1}(0,1)$ such that

$$
F(t, x) \geq-c(t)\left(1+|x|^{l}\right)
$$

for almost every $t \in[0,1]$ and for all $x \in R$.

Then, problem (1) has a solution.

Proof. $\left(A_{3}\right)$ implies that for each $x \in W$

$$
\begin{gathered}
\left(x(0), x\left(t_{1}\right), x(1)\right) A\left(x(0), x\left(t_{1}\right), x(1)\right)^{T} \\
\geq k_{1}\left(x^{2}(0)+x^{2}\left(t_{1}\right)+x^{2}(1)\right) .
\end{gathered}
$$

By (9), (32), and $\left(A_{4}\right)$,

$$
\begin{aligned}
\phi(x) \geq & \frac{1}{2}\|(I-P) x\|^{2}-\int_{0}^{1} c(t)\left(|x(t)|^{l}+1\right) d t \\
& +\frac{1}{2} k_{1}\left(x^{2}(0)+x^{2}\left(t_{1}\right)+x^{2}(1)\right) \\
\geq & \frac{1}{2} k_{3}\|x\|^{2}-\int_{0}^{1} c(t) d t\left(2\|x\|^{l}+1\right),
\end{aligned}
$$

where $k_{3}=\min \left\{k_{1}, 1\right\}$. It follows that $\phi(x) \rightarrow+\infty$ as $\|x\| \rightarrow$ $\infty$. Thus, $\phi$ has a critical point and problem (1) has a solution.

Theorem 3. Assume that $\left(A_{1}\right),\left(A_{2}\right)$, and $\left(A_{3}\right)$ are satisfied. Assume also that the following conditions hold:

$\left(A_{4}^{\prime}\right)$ there is a positive function $c \in L^{1}(0,1)$ such that

$$
F(t, x) \geq-c(t)\left(1+x^{2}\right)
$$

for almost every $t \in[0,1]$ and for all $x \in R$.
$\left(A_{5}\right) 4 \int_{0}^{1} c(t) d t<k_{3}$, where $k_{3}$ is in the proof of Theorem 2.

Then, problem (1) has a solution.

Proof. This proof is similar to the proof of Theorem 2.

Theorem 4. Assume that $\left(A_{1}\right)-\left(A_{4}\right)$ are satisfied. Assume also that the following condition holds:

$\left(A_{6}\right)$ there are two positive constants $d_{1}$ and $d_{2}$ with $d_{1}<$ $\sqrt{6} d_{2}$ such that

$$
\begin{gathered}
\int_{0}^{1} F\left(t, d_{2}\right) d t<-\frac{3 k_{2}}{2} d_{2}^{2}, \\
\int_{0}^{1} \max _{|x| \leq \sqrt{k_{4}} d_{1}}[-F(t, x)] d t<\frac{k_{1}}{4} d_{1}^{2},
\end{gathered}
$$

where $k_{4}=\max \left\{k_{1}, 1\right\}$.

Then, problem (1) has at least three solutions.

In order to prove this theorem, we need the following theorem (see Theorem 2.1 of [18]).

Theorem A. Let $X$ be a reflexive real Banach space; let $\Phi$ : $X \rightarrow R$ be a sequentially weakly lower semicontinuous, coercive, and continuously Gâteaux differentiable functional whose Gâteaux derivative admits a continuous inverse on $X^{*}$; and let $\Psi: X \rightarrow R$ be a sequentially weakly upper semicontinuous and continuously Gâteaux differentiable functional whose Gâteaux derivative is compact. Assume that there exist $r \in R$ and $x_{0}, \bar{x} \in X$, with $\Phi\left(x_{0}\right)<r<\Phi(\bar{x})$ and $\Psi\left(x_{0}\right)=0$, such that

(i) $\sup _{\Phi(x) \leq r} \Psi(x)<\left(r-\Phi\left(x_{0}\right)\right)\left(\Psi(\bar{x}) /\left(\Phi(\bar{x})-\Phi\left(x_{0}\right)\right)\right)$

(ii) for each $\left.\lambda \in \Lambda_{r}:=\right]\left(\Phi(\bar{x})-\Phi\left(x_{0}\right)\right) / \Psi(\bar{x}),(r-$ $\left.\Phi\left(x_{0}\right)\right) / \sup _{\Phi(x) \leq r} \Psi(x)$ [ the functional $\Phi-\lambda \Psi$ is coercive.

Then, for each $\lambda \in \Lambda_{r}$ the functional $\Phi-\lambda \Psi$ has at least three distinct critical points in $X$.

Proof of Theorem 4. Let $X=W$ and define

$$
\begin{aligned}
\Phi(x)= & \frac{1}{2}\|(I-P) x\|^{2} \\
& +\frac{1}{2}\left(x(0), x\left(t_{1}\right), x(1)\right) A\left(x(0), x\left(t_{1}\right), x(1)\right)^{T}
\end{aligned}
$$

for each $x \in X$. Then, $\Phi$ is a sequentially weakly lower semicontinuous and continuously differentiable functional whose derivative is given by

$$
\begin{aligned}
\left(\Phi^{\prime}(x), y\right)= & ((I-P) x,(I-P) y) \\
& +\left(x(0), x\left(t_{1}\right), x(1)\right) A\left(y(0), y\left(t_{1}\right), y(1)\right)^{T}
\end{aligned}
$$


for all $y \in X$. It follows from (36) that for each $x \in W$,

$$
\begin{aligned}
\Phi(x) \geq & \frac{1}{2}\|(I-P) x\|^{2} \\
& +\frac{1}{2} k_{1}\left[x^{2}(0)+x^{2}\left(t_{1}\right)+x^{2}(1)\right] \geq \frac{1}{2} k_{3}\|x\|^{2},
\end{aligned}
$$

so that $\Phi$ is coercive. Because $I-\Phi^{\prime}$ is a compact operator, $\Phi^{\prime}$ has the continuous inverse if and only if 0 is not the eigenvalues of $\Phi^{\prime}$. If 0 is the eigenvalues of $\Phi^{\prime}$ and $\beta(t)$ is a eigenvector of $\Phi^{\prime}$ associated with the eigenvalue 0 , then (37) implies that

$$
\begin{aligned}
0= & \left(\Phi^{\prime}(\beta), \beta\right) \\
= & \|(I-P) \beta\|^{2} \\
& +\left(\beta(0), \beta\left(t_{1}\right), \beta(1)\right) A\left(\beta(0), \beta\left(t_{1}\right), \beta(1)\right)^{T} \\
\geq & k_{3}\|\beta\|^{2}>0 .
\end{aligned}
$$

This is a contradiction, and hence $\Phi^{\prime}$ has the continuous inverse. Set $\Psi(x)=-\int_{0}^{1} F(t, x(t)) d t$ for $x \in X$. Then, $\Psi$ is a sequentially weakly upper semicontinuous and continuously differentiable functional whose derivative is compact.

Setting $x_{0}=0$ and $\bar{x}=d_{2}$ for all $t \in[0,1]$, then $\Phi\left(x_{0}\right)=$ $\Psi\left(x_{0}\right)=0$ and

$$
\frac{3}{2} k_{2} d_{2}^{2} \geq \Phi(\bar{x})=\frac{1}{2}\left(d_{2}, d_{2}, d_{2}\right) A\left(d_{2}, d_{2}, d_{2}\right)^{T} \geq \frac{3}{2} k_{1} d_{2}^{2},
$$

since $(I-P) \bar{x}=0$. Setting $r=(1 / 4) k_{1} d_{1}^{2}$, by $d_{1}<\sqrt{6} d_{2}$ and (40), we obtain

$$
\Phi\left(x_{0}\right)=0<r<\frac{3}{2} k_{1} d_{2}^{2} \leq \Phi(\bar{x}) .
$$

By (38), $\Phi(x) \leq r$ implies that $|x(t)| \leq \sqrt{k_{4}} d_{1}$ for every $t \in$ $[0,2 \tau]$ since $k_{1}=k_{3} k_{4}$. From $\left(A_{6}\right)$ and (40), we have

$$
\begin{aligned}
\sup _{\Phi(x) \leq r} \Psi(x) & \leq \int_{0}^{1} \max _{|x| \leq \sqrt{k_{4}} d_{1}}[-F(t, x)] d t \\
& <\frac{k_{1}}{4} d_{1}^{2}<\frac{k_{1} d_{1}^{2} \int_{0}^{1}\left[-F\left(t, d_{2}\right)\right] d t}{6 k_{2} d_{2}^{2}} \\
& \leq(r-\Phi(0)) \frac{\Psi(\bar{x})}{\Phi(\bar{x})-\Phi(0)},
\end{aligned}
$$

and (i) in Theorem A holds.

Since

$$
\begin{aligned}
\frac{\Phi(\bar{x})-\Phi\left(x_{0}\right)}{\Psi(\bar{x})} & \leq \frac{(3 / 2) k_{2} d_{2}^{2}}{\int_{0}^{1}\left[-F\left(t, d_{2}\right)\right] d t}<1 \\
& <\frac{(1 / 4) k_{1} d_{1}^{2}}{\int_{0}^{1} \max _{|x| \leq \sqrt{k_{4}} d_{1}}[-F(t, x)] d t} \\
& \leq \frac{r-\Phi\left(x_{0}\right)}{\sup _{\Phi(x) \leq r} \Psi(x)},
\end{aligned}
$$

we can take $\lambda=1$ in Theorem A. Therefore, it is easy to show that

$$
(\Phi-\Psi)(x) \geq \frac{1}{2} k_{3}\|x\|^{2}-\int_{0}^{1} c(t) d t\left(2\|x\|^{l}+1\right),
$$

so that $\Phi-\Psi$ is coercive. Using Theorem A, problem (1) has at least three solutions.

\section{Conflict of Interests}

The author declares that there is no conflict of interests regarding the publication of this paper.

\section{Acknowledgment}

This work is supported by the Natural Science Foundation of Shandong Province of PR China (ZR2011AL007).

\section{References}

[1] G. Bao, X. Xu, and Y. Song, "Positive solutions for three-point boundary value problems with a non-well-ordered upper and lower solution condition," Applied Mathematics Letters, vol. 25, no. 4, pp. 767-770, 2012.

[2] J. Zhao and W. Ge, "A necessary and sufficient condition for the existence of positive solutions to a kind of singular three-point boundary value problem," Nonlinear Analysis: Theory, Methods and Applications, vol. 71, no. 9, pp. 3973-3980, 2009.

[3] F. Li, M. Jia, X. Liu, C. Li, and G. Li, "Existence and uniqueness of solutions of second-order three-point boundary value problems with upper and lower solutions in the reversed order," Nonlinear Analysis: Theory, Methods and Applications, vol. 68, no. 8, pp. 2381-2388, 2008.

[4] F. Li, J. Sun, and M. Jia, "Monotone iterative method for the second-order three-point boundary value problem with upper and lower solutions in the reversed order," Applied Mathematics and Computation, vol. 217, no. 9, pp. 4840-4847, 2011.

[5] M. Pei and S. K. Chang, "The generalized quasilinearization method for second-order three-point boundary value problems," Nonlinear Analysis: Theory, Methods and Applications, vol. 68, no. 9, pp. 2779-2790, 2008.

[6] M. K. Kwong and J. S. W. Wong, "Solvability of second-order nonlinear three-point boundary value problems," Nonlinear Analysis: Theory, Methods and Applications, vol. 73, no. 8, pp. 2343-2352, 2010.

[7] X. Han, "Positive solutions for a three-point boundary value problem at resonance," Journal of Mathematical Analysis and Applications, vol. 336, no. 1, pp. 556-568, 2007.

[8] B. Liu, L. Liu, and Y. Wu, "Positive solutions for a singular second-order three-point boundary value problem," Applied Mathematics and Computation, vol. 196, no. 2, pp. 532-541, 2008.

[9] J. Li and J. Shen, "Positive solutions for three-point boundary value problems for second-order impulsive differential equations on infinite intervals," Journal of Computational and Applied Mathematics, vol. 235, no. 8, pp. 2372-2379, 2011.

[10] S. Liang and J. Zhang, "The existence of countably many positive solutions for some nonlinear singular three-point impulsive boundary value problems," Nonlinear Analysis: Theory, Methods and Applications, vol. 71, no. 10, pp. 4588-4597, 2009. 
[11] T. Jankowski, "Positive solutions of three-point boundary value problems for second order impulsive differential equations with advanced arguments," Applied Mathematics and Computation, vol. 197, no. 1, pp. 179-189, 2008.

[12] H. Sun, Y. Li, J. J. Nieto, and Q. Tang, "Existence of solutions for Sturm-Liouville boundary value problem of impulsive differential equations," Abstract and Applied Analysis, vol. 2012, Article ID 707163, 19 pages, 2012.

[13] J. J. Nieto and D. O’Regan, "Variational approach to impulsive differential equations," Nonlinear Analysis: Real World Applications, vol. 10, pp. 680-690, 2009.

[14] Y. Tian and W. Ge, "Variational methods to Sturm-Liouville boundary value problem for impulsive differential equations," Nonlinear Analysis: Theory, Methods and Applications, vol. 72, no. 1, pp. 277-287, 2010.

[15] Y. Tian and W. Ge, "Multiple solutions of impulsive Sturm-Liouville boundary value problem via lower and upper solutions and variational methods," Journal of Mathematical Analysis and Applications, vol. 387, no. 2, pp. 475-489, 2012.

[16] H. Zhang and Z. Li, "Variational approach to impulsive differential equations with periodic boundary conditions," Nonlinear Analysis: Real World Applications, vol. 11, no. 1, pp. 67-78, 2010.

[17] J. Mawhin and M. Willem, Critical Point Theory and Hamiltonian Systems, Springer, New York, NY, USA, 1989.

[18] G. Bonanno and G. Riccobono, "Multiplicity results for SturmLiouville boundary value problems," Applied Mathematics and Computation, vol. 210, no. 2, pp. 294-297, 2009. 


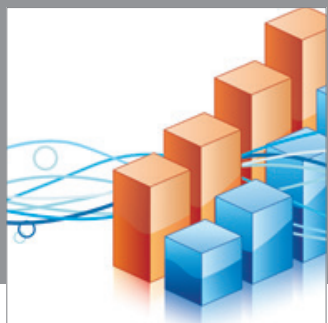

Advances in

Operations Research

mansans

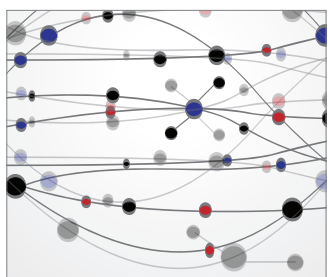

The Scientific World Journal
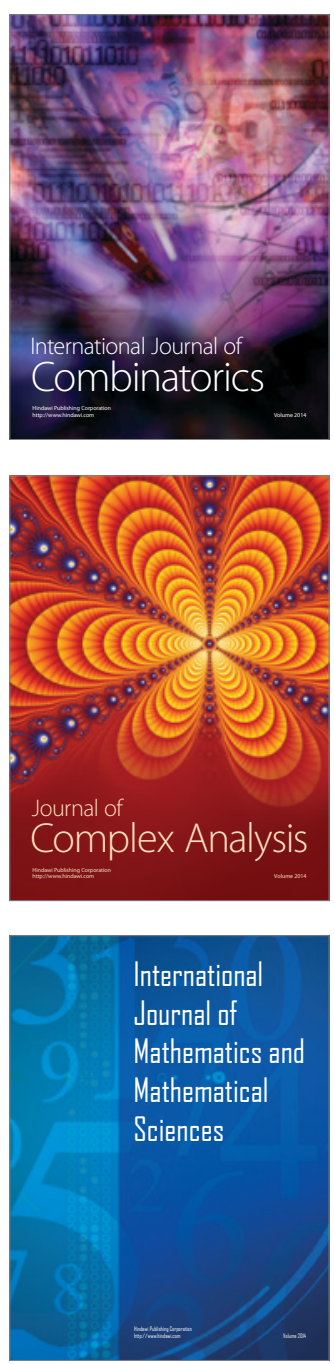
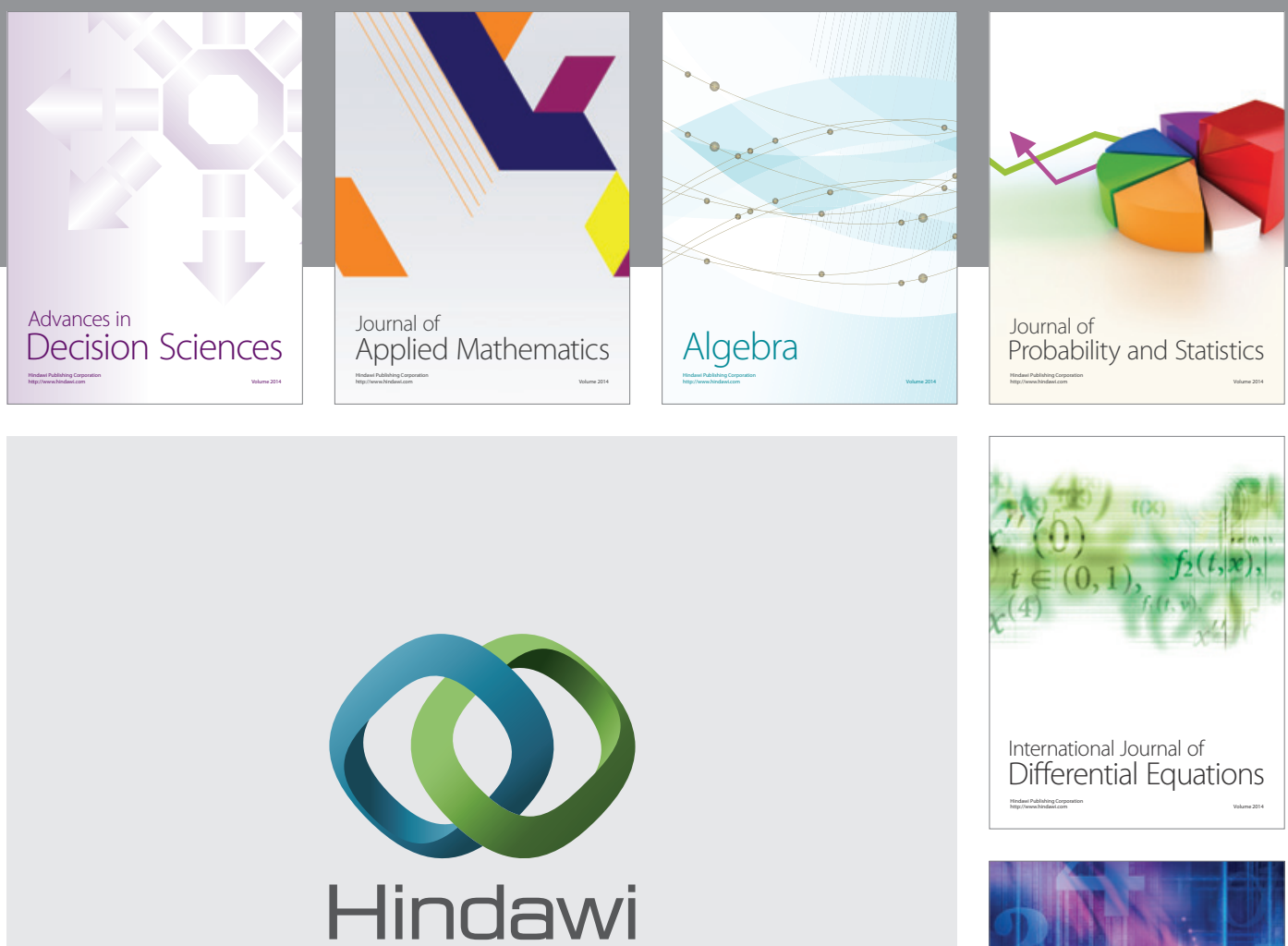

Submit your manuscripts at http://www.hindawi.com


Journal of

Function Spaces

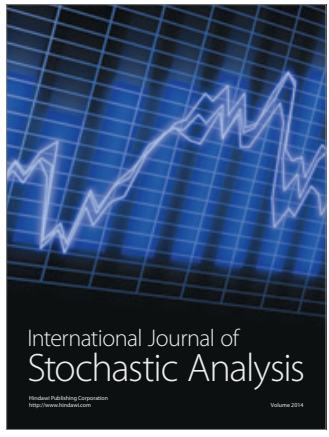


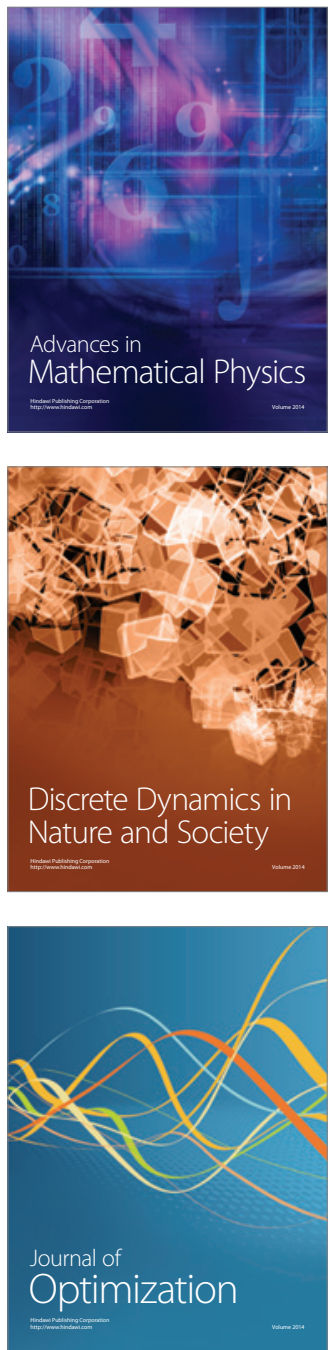